\title{
Prehospital Health Care Management Platform
}

\author{
E.Kyriacou, S. Christou, \\ G. Hadjichristofi \\ Dep. of Comp. Science and \\ Eng. Frederick University, \\ Limassol, Cyprus \\ e.kyriacou@frederick.ac.cy
}

\author{
R. Constantinou \\ Ambulance Services \\ Department, Ministry of \\ Health, \\ Nicosia, Cyprus
}

\author{
A.Panayides \\ Dep. of Electrical and \\ Electronics Engineering, \\ Imperial College, \\ London, UK
}

\author{
C. Pattichis \\ Dept. of Computer Science, \\ University of Cyprus, \\ Nicosia, Cyprus
}

\begin{abstract}
Through this work we had created a platform that will support the emergency health care procedures starting from an emergency call until a patient is transferred to a central hospital. The platform consists of a) a system that supports emergency dispatch protocols, b) data recording and workflow management of ambulance department and c) an emergency telemedicine system which can support patient handling from expert personnel. Technically the system is finished and is currently under technical verification process before moving to a clinical pilot phase.
\end{abstract}

Keywords-Emergency health care suport, emergency workflow management, emergency telemedicine

\section{INTRODUCTION}

The accurate management of data interchanged between an ambulance vehicle and a hospital emergency department can become a useful tool which can help paramedics when handling an emergency incident as well as the emergency department that will handle the patient when transferred to a central hospital. Along these steps we had tried to record all the procedures of the Ambulance Department of the Ministry of Health - Cyprus and create a system which will be able to support Pre hospital emergency care.

\section{METHODOLOGY}

The system will be installed at the ambulance response centre in order to support the procedure followed from the time that a call is received until the patient is transferred to a main hospital [1]. The main operational features of the system are:

- Support emergency dispatchers when deciding using the Emergency dispatch protocols

- Live monitoring of Ambulance vehicles status and incident involved

- Real time data exchange of Ambulance vehicles and call centre

oEach vehicle will be equipped with a 7 inch android tablet pc. Through which paramedics can get information about the incident and can send information back to the control centre through $3 \mathrm{G}$ network

- Live monitoring of patients at incident scene and while in transport through a portable telemedicine unit. The unit can transmit vital biosignals and video from the scene[2]

- All information is available at First aid control center and on call doctors. This is going to help in the decision for in hospital patient handling ie. Support cardiac clinic decision for patients needing emergency angioplasty.

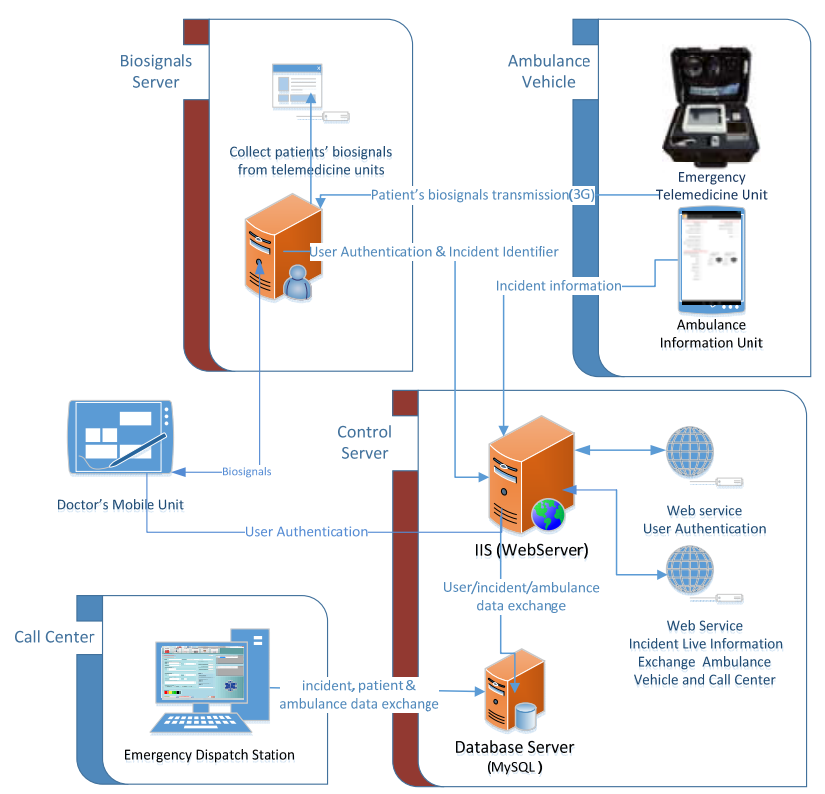

Figure 1. Prehospital care platform general architecture

The General architecture of the system is shown in figure 1. The communication between the mobile units and the hospital units is being done using TCP/IP over mobile 3G networks. Data from Ambulances to the server are exchanged using web services on an IIS web server. The access to the system is enabled using unique id for each unit SSL for communication and different access levels depending on the user group.

Concluding, through these are the initial steps in order to create an electronic system that will support the Ambulance department of the ministry of health of Cyprus which is currently going through a restructuring phase

\section{REFERENCES}

[1] P. Constantinides and M. Barret, "A Narrative Approach to Understanding Coordination Practices in Emergency Response," Information \& Organization, vol. 22, pp. 273-294, 2012.

[2] A. Panayides, Z. Antoniou, Y. Mylonas, M. S. Pattichis, A. Pitsillides, and C. S. Pattichis, "High-resolution, low-delay, and error-resilient medical ultrasound video communication using H.264/AVC over mobile WiMAX networks,” IEEE J. Biomed. Health Informat., vol. 17, no. 3, pp. 619-628, May 2013. 